\title{
Simultaneous and Proportional Myocontrol of a Hand Exoskeleton for Spinal Muscular Atrophy: a preliminary evaluation
}

\author{
Marco Ricciardi, Alberto Topini, Nicola Secciani, Alessandro Ridolfi, Claudio Castellini
}

\begin{abstract}
Spinal Muscular Atrophy (SMA) is a neuromuscular disease characterized by the degeneration of the $\alpha$-motor neurons in the spinal cord, resulting in progressive proximal muscle weakness and paralysis. It is the second most common fatal autosomal recessive disorder after cystic fibrosis in the world. In the context of assistive robotics for SMA, in this work the authors have preliminarily assessed the feasibility of using low-cost electromyography pattern recognition and simultaneous/proportional myocontrol to enforce smooth, intuitive control of an assistive hand exoskeleton system. A target achievement control test has involved ten healthy subjects. Synthetic noise has been added to their surface ElectroMyoGraphic (sEMG) signals in order to reach a signal-to-noise ratio similar to that of sEMG signals gathered from a SMA patient. The results indicate that, even neglecting any learning effect, an SMA patient could reach an average success rate of up to $82 \%$ through the proposed approach.
\end{abstract}

\section{INTRODUCTION}

Spinal Muscular Atrophy (SMA) is a severe neuromuscular disease characterized by the degeneration of $\alpha$-motor neurons in the spinal cord, resulting in a progressive proximal muscle weakness and paralysis [1]. Although there currently is no treatment for SMA, much can be done in the context of Assistive Robotics to assist the patient's loss of motor functions. In particular, given the type of SMA and its stage, the patient can still be able to produce significant voluntary muscular activity. Indeed, as long as the patient can produce reasonably distinct and repeatable sEMG signal patterns, Machine Learning (ML) can be used to control an assistive device. Given the nature of the interaction between the devices and their users, it is desirable to let the patient take full, proportional, smooth and intuitive control [2].

Two different ML approaches [3], [4] are currently being tested by the authors to assess the control experience on an assistive Hand Exoskeleton System (HES) [5]. In this work, the feasibility of applying simultaneous, proportional and incremental myocontrol to the device has been evaluated through a Target Achievement Control (TAC) test [6], simulated by means of a Blender model of the HES. At this point of the experimentation, the HES has no other purpose than to visually familiarize the user with the final system.

This work was partially supported by the German Research Society project Deep-Hand (DFG Sachbeihilfe CA-1389/1-2).

The authors would like to thank Annette Hagengruber and Jörn Vogel of the DLR for useful insights and for making available to us sEMG signals recorded from a SMA patient.

M.Ricciardi, A. Topini, N. Secciani, and A. Ridolfi are with the Department of Industrial Engineering (DIEF), University of Florence, Via di Santa Marta 3, 50139, Florence, Italy

C. Castellini is with the Institute of Robotics and Mechatronics, DLR German Aerospace Center, 82234 Wessling, Germany

Corresponding author contact: alberto.topinieunifi.it

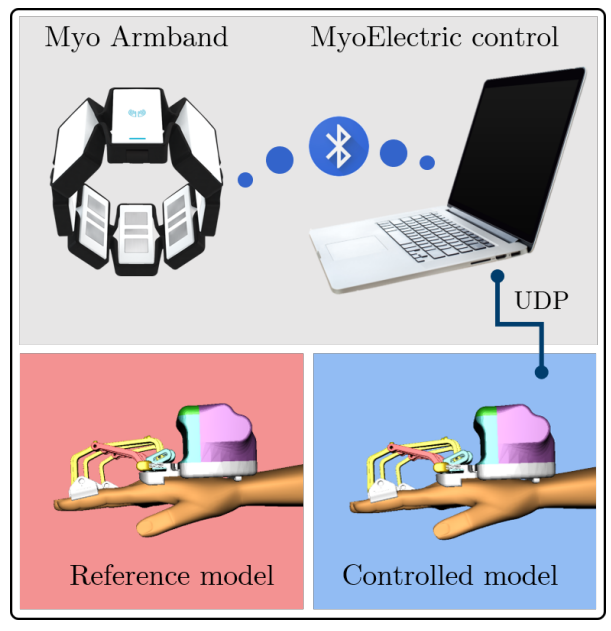

Fig. 1. The exploited experimental setup. On the gray background the employed hardware, while below the virtual models of the HES.

\section{MAterials AND Methods}

In this study, ten healthy participants (aged between 24 and 61 years) — voluntarily enrolled - have been asked to control a virtual avatar of the exoskeleton using their own voluntary muscle activity, recorded in real-time using a Myo armband. Synthetic noise (Gaussian noise with variable variance) has been added to the sEMG signals in order to progressively reach the Signal-to-Noise Ratio (SNR) that characterized those collected from SMA patients. It is worth noting that, although there are no specific references in the literature, this approach has been considered sufficiently reliable since SMA is known to weaken the muscle activity (thus lowering the SNR) without altering neuromuscular patterns. The target SNR (SNR4 = 15.97) has been identified through previous recordings from a patient affected by SMA type II, and other three different SNR levels have been defined $($ SNR3 = 52.80; SNR2 = 125.11; SNR1 = 211.82); where a higher SNR corresponds to a better signal.

\section{A. Experimental Setup}

As can be seen from Fig. 1, the experimental setup relies on the wearing of the Myo armband that transmits the sEMG signal, by means of Bluetooth communication, to a laptop running the intent detection system. The sEMG signal is acquired, processed and labeled by a custom software suite written in the $\mathrm{C \#}$ and Python languages. Finally, the HES configuration, reference and prediction, are provided to two distinct Blender digital twins through a User Datagram Protocol (UDP) message-based system. 


\section{B. Experimental Protocol}

The test participants have been asked to wear the Myo armband in a preliminary stage of the experiment as well as to avoid sensor doffing or displacements; as a matter of fact, this procedure apriostically reduces the presence of undesired effects, such as electrode shifts, and allows for coherent results over the whole experimental campaign. The procedural architecture comprises of two primary hierarchical stages: firstly, during the so-called "training phase", the myoelectric control develops and learns a regression model that deals with the prediction of the hand configurations starting from the sEMG signals; subsequently, the TAC test evaluates the performance of the learned model to generalize over several, distinct tasks. For what concerns the data acquisition and training process, the subjects, after having received a comprehensive description of the routines, carry out three different hand gestures (complete closure, complete extension, and resting) in a double-repetition sequence. The whole procedure exploits a straightforward Graphical User Interface (GUI) which, by guiding the test contributors, uniforms the test architecture over the whole set of participants.

Conversely, the TAC test has been structured so as to achieve four actions (complete closure, semi-closure, semiextension, complete extension) alongside four incremental SNR levels, for a total of sixteen different combinations. The participants have been then asked to fulfill five unordered repetitions of each of the aforementioned combinations: the whole test was hence composed of series of 80 tasks.

As far as the specific protocol is concerned, the participants have been requested to reproduce and hold for $1.5 \mathrm{~s}$ the hand configuration displayed by the "reference model" within a maximum time interval of $20 \mathrm{~s}$. Since the predicted hand motion is also shown by the "controlled model", matching the positions of the two virtual avatars emerges as the actual aim for the user during the test.

\section{RESULTS}

The above-described procedure has been quantitatively evaluated by means of two major metrics: the Success Rate (SR), defined as the percentage of correctly performed tasks

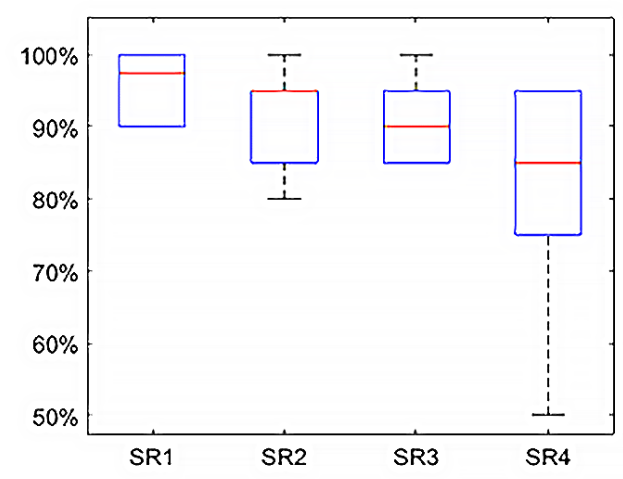

Fig. 2. Boxplot reporting the Success Rate for SNR level. The numerical index of the SR on the x-axis indicates the corresponding SNR level. with respect to the total number of achievable tasks, and the Time to Complete the Task (TCT), which outlines the time required to successfully fulfill a task. As illustrated in Fig. 2 , the SR average value decreases, within a range $82 \div 96 \%$, as the noise level rises; additionally, the TCT outcomes do confirm this performance-worsening pattern (Table I) by highlighting larger TCT for noisier sEMG signals.

TABLE I

\begin{tabular}{cc}
$\begin{array}{r}\text { TCT OVER DECREASING SNR LEVELS } \\
\text { SNR level }\end{array}$ & TCT [s] \\
\hline SNR1 & $4.217 \pm 1.535$ \\
SNR2 & $4.414 \pm 1.924$ \\
SNR3 & $5.597 \pm 2.256$ \\
SNR4 & $5.614 \pm 2.367$
\end{tabular}

\section{Discussion AND CONCLUSIONS}

On average, our participants have reached a TAC test SR ranging from $82 \%$ to $96 \%$, depending on the SNR level. An SR of $82 \%$ corresponds to the lowest SNR, which was akin to that found on the signals generated by the reference SMA patient. With all due caution, this could possibly indicate that a patient of a similar type and stage of SMA could achieve similar results, using the same myocontrol system while controlling the HES. Notice, moreover, that the tests were quite short (about 20 minutes per participant), so no learning effect whatsoever has been observable either in their SRs or in their TCTs. It seems reasonable to claim that, if a patient were allowed to wear and control the exoskeleton for a longer time and across multiple sessions, he/she would display a definite learning curve, thereby further improving the results. Finally it is important to notice that, even if the model training has been performed over just three different gestures, the regression algorithm allows for the continouos discrimination of all the intermediate positions.

\section{REFERENCES}

[1] A. D'Amico, E. Mercuri, F. D. Tiziano, and E. Bertini, "Spinal muscular atrophy," Orphanet journal of rare diseases, vol. 6, no. 1, pp. 1-10, 2011.

[2] P. Beckerle, C. Castellini, and B. Lenggenhager, "Robotic interfaces for cognitive psychology and embodiment research: a research roadmap," Wiley Interdisciplinary Reviews: Cognitive Science, vol. 10, no. 2, p. e1486, 2019.

[3] N. Secciani, M. Bianchi, A. Ridolfi, F. Vannetti, and B. Allotta, "Assessment of a hand exoskeleton control strategy based on user's intentions classification starting from surface EMG signals," in International Symposium on Wearable Robotics, January 2019, pp. 440-444.

[4] I. Strazzulla, M. Nowak, M. Controzzi, C. Cipriani, and C. Castellini, "Online bimanual manipulation using surface electromyography and incremental learning," IEEE Transactions on Neural Systems and Rehabilitation Engineering, vol. 25, no. 3, pp. 227-234, 2016.

[5] N. Secciani, M. Bianchi, A. Ridolfi, F. Vannetti Yary Volpe, L. Governi, M. Bianchini, and B. Allotta, "Tailor-made hand exoskeletons at the university of florence: From kinematics to mechatronic design," Machines, vol. 7, no. 2, p. 22, April 2019.

[6] A. M. Simon, L. J. Hargrove, B. A. Lock, and T. A. Kuiken, "The target achievement control test: Evaluating real-time myoelectric pattern recognition control of a multifunctional upper-limb prosthesis," Journal of rehabilitation research and development, vol. 48, no. 6, p. 619, 2011. 